\section{RELIABLE HANDPIECE REPAIRS}

Handpiece Express is the brand name for The Dental Directory's handpiece repairs and servicing branch, offering service and repair packages for all handpieces, motors, couplings and sonic scalers.

Every repair and service by

Handpiece Express is guaranteed, with each instrument tested to ensure its performance matches or exceeds original manufacturer specifications. Routine tests include: speed, turbine balance, concentricity and autochuck pull force. Handpiece Express

technicians will also run a series of tests using a diagnostic acoustic analyser to ensure optimum handpiece performance.

All Handpiece Express technicians are manufacturer-accredited, and will ensure the quality of every repair by individually inspecting, testing and certifying all replacement parts. As a customer, you will also be able to speak personally with the technician undertaking your particular repair, and if your handpiece is not returned to you within 24 hours of an approved telephone quote, you will not be charged.

Reader response number 58

\title{
INTUITIVE DESIGN AND FUNCTIONALITY
}

Heka Dental's UNIC Treatment Centres will inspire you to become the creative genius you have always aspired to be.

Heka Dental's bespoke UNIC

Treatment Centres combine aesthetic beauty with state-of-the-art ergonomic efficiency. Now available in an even wider range of colours, UNIC is available in the UK from DB Dental and Axis Dental Equipment.

The ultimate embodiment of feedback from patients, dentists and service engineers, UNIC's inviting appearance and carefully thought-through functionality creates the perfect environment for a pleasant dental visit. Designed by David Lewis, UNIC is the epitomy of ergonomic design. Everything - instruments, trays, light, $\mathrm{X}$-ray unit - is within easy reach. Everything is exactly where you would expect it to be, making even complex clinical procedures easier, more efficient and comfortable for the patient and dental team.

With a fully comprehensive threeyear warranty, every component is manufactured by Heka Dental in order to ensure the highest possible quality. Reader response number 59

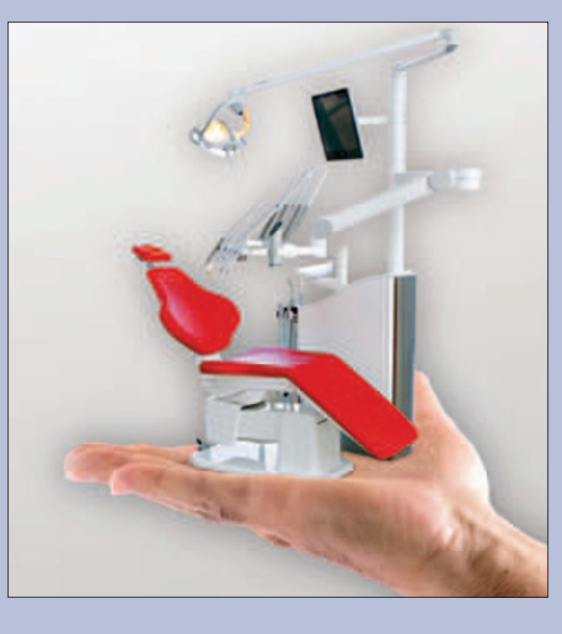

IMAGING AT YOUR FINGERTIPS

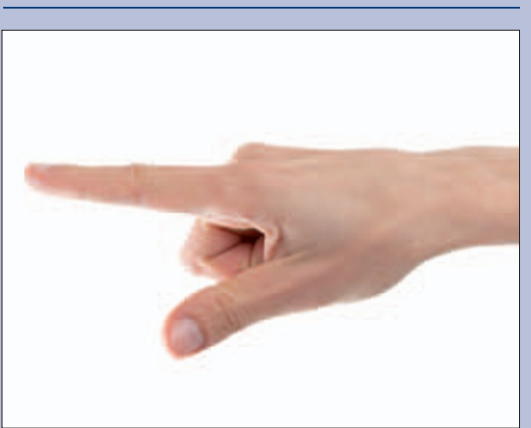

Incorporating the latest 3D imaging technology, Sident Dental Systems' new Orthophos XG 3D delivers excellent image quality, minimum radiation dose, intuitive software and efficient clinical workflow. It enables the operator to switch between 2D panoramic and 3D imaging at the touch of a button. Optimised for day-to-day practice assignments it incorporates height adjustable field of view options for maximum efficiency. It enables clinicians to see more detail and treat patients more efficiently because it provides more diagnostic benefits and better treatment planning capabilities.

Its 3D capabilities are ideal for implant procedures enabling implantologists to generate radiographs, carry out in-depth diagnostics, treatment plan and order surgical guides ... ultimately enabling them to achieve greater diagnostic accuracy and place more implants 'in-house'.

Similarly orthodontists can use a cephalometric version to benefit from its high quality traditional 2D imaging to identify unerupted teeth and its 3D capacity to optimise treatment planning. This module can be retro-fitted when desired.

CEREC users can use their

CEREC design data for simultaneous prosthetic and surgical implant planning.

Whichever option is most appropriate the fully interactive Easypad touch interface facilitates perfect workflow and puts the entire spectrum of imaging programs at the user's fingertips.

Reader response number 61 of all of your VLC materials.

With a high intensity, narrow spectrum of light, the G-Light emits the maximum amount of light energy rechargeable light can be used over 400 times for ten seconds without any reduction in intensity. The constant light energy guarantees efficient and deep curing every time.

This lightweight, ergonomically designed, cordless curing light can be set to a range of practical curing times, as well as continuous mode.

Reader response number 60 